\title{
Gazing into the crystal ball: future considerations for ensuring sustained growth of the functional food and nutraceutical marketplace
}

\author{
Christopher P. F. Marinangeli ${ }^{1}$ and Peter J. H. Jones ${ }^{2 *}$ \\ ${ }^{1}$ Kellogg Canada Inc., 5350 Creekbank Road, Mississauga, Ontario, Canada, L4W 551 \\ ${ }^{2}$ Richardson Centre for Functional Foods and Nutraceuticals, University of Manitoba, 196 Innovation Drive, Winnipeg, \\ Manitoba, Canada, R3T 2N2
}

\begin{abstract}
Over the last decade the concept of functional foods and nutraceuticals (FFN) has gained support from various stakeholders including the food industry, scientific and academic community, government institutions or regulators, producers and consumers. However, as one begins to evaluate the global FFN industry, several issues emerge including (i) a lack of consensus across jurisdictions for acknowledging safe and efficacious FFN, (ii) challenges regarding the classification of novel food-derived bioactives as FFN or drugs, and (iii) a disconnect between nutrient requirements and dosages of FFN required to facilitate health benefits. The objectives of the present review are to discuss the role of existing stakeholders within the FFN marketplace and identify performance indicators for growth within the FFN sector. In addition, the following report provides feasible resolutions to present and future challenges facing the global FFN industry to ensure sustained long-term growth.
\end{abstract}

Key words: Functional foods: Nutraceuticals: Regulatory agencies: Policy: Drugs

\section{Introduction}

Although definitions may differ across jurisdictions, a functional food is typically defined as a food that is consumed as part of a usual diet that is demonstrated to have physiological benefits and/or reduce the risk of chronic disease beyond providing basic nutritional functions ${ }^{(1)}$. Basic nutritional functions encompass a nutrient's contribution(s) to normal functions or biological activities of the body such as $\mathrm{Ca}$ and bone formation, fibre and laxation or protein and tissue building/repair. In Australia, the National Centre of Excellence for Functional Foods proposed that the definition of functional foods be expanded to encompass 'those (foods) promoted on a health platform and based on scientific evidence ${ }^{,(2)}$. Nutraceuticals represent bioactive constituents of functional foods that facilitate a physiological benefit or provide protection against chronic disease when provided in caplet or pill format ${ }^{(1)}$. Although nutraceuticals can be made available as supplements, breakthroughs in food technologies permit the addition of isolated bioactives to food matrices that were previously devoid of the added nutraceutical. Similarly, novel food technologies can be utilised to enhance the concentration of a bioactive compound within its native food. These approaches can transform regular foods into functional foods.

Over the past two decades the concept of functional foods and nutraceuticals (FFN) has gained support from various stakeholders including the food industry, scientific and academic communities, government institutions or regulators, producers and consumers. Combined, the aforementioned stakeholders have established a strong FFN industry that grew by US\$50.9 billion from 2000 to $2007^{(3,4)}$ with the mandate to reduce the incidence of chronic disease. Nonetheless, lack of consensus across jurisdictions for acknowledging safe and efficacious FFN, challenges regarding the classification of novel foodderived bioactives as FFN or drugs, and the presence of a disconnect between nutrient requirements and dosages of FFN required to facilitate a health benefit, represent hurdles that need to be addressed to ensure sustained growth of the global FFN industry.

The objectives the present review are therefore to identify the independent and interactive roles of existing stakeholders within the FFN marketplace, assess performance indicators for growth within the FFN sector, and

Abbreviations: DRI, dietary reference intake; EFSA, European Food Safety Authority; FFN, functional foods and nutraceuticals; LDL-C, LDL-cholesterol; PS, plant sterols/stanols.

*Corresponding author: Dr Peter J. H. Jones, fax +1 204474 7552, email peter_jones@umanitoba.ca 
discuss factors that will ensure that the FFN industry continues to grow as well as deliver appreciable health benefits to the population.

\section{Existing stakeholders within the functional foods and nutraceuticals marketplace}

The FFN marketplace possesses multi-sectorial representation, including the following stakeholders: industry, scientists, regulators, producers and consumers (Table 1). The success or failure of the FFN marketplace is dependent on the ability of these stakeholders to work cooperatively and support each other's interests, objectives, opportunities and weaknesses. The following section will discuss the role of each stakeholder within the FFN arena.

\section{Industry}

Continued profitability within the FFN market is demonstrated by its consistent annual growth. From 2000 to 2007, the global FFN industry experienced 50\% growth in sales from US\$102.1 billion to US\$153 billion per year, respectively $^{(3,4)}$. Here, the USA, Europe and Japan are the world's largest markets at 34, 28 and $21 \%$, respectively ${ }^{(4)}$. However, other jurisdictions including China, Canada and Australia/New Zealand continue to show rapid growth within the FFN industry. As consumers continue to take a vested interest in their own health and wellbeing, FFN companies continue to explore and market efficacious foods, ingredients and supplements aimed at preventing chronic disease.

Table 1. Summary of stakeholder functions within the global functional food industry

\begin{tabular}{ll}
\hline Stakeholder & Function \\
\hline Industry & $\begin{array}{l}\text { Fund clinical trials that explore functional foods and } \\
\text { nutraceutical efficacy, effectiveness and safety } \\
\text { Provide food to consumers that incorporate effica- } \\
\text { cious levels of bioactives that reduce risk factors } \\
\text { for disease } \\
\text { Design and conduct robust clinical and preclinical } \\
\text { studies that demonstrate efficacy and safety as well } \\
\text { as delineate mechanisms by which foods and/or } \\
\text { bioactives reduce risk factors for disease } \\
\text { Key opinion leaders that serve as liaisons of reliable } \\
\text { science to industry, regulators and consumers } \\
\text { Implement and enforce laws surrounding the sale and } \\
\text { health claim messages for functional foods and } \\
\text { food ingredients } \\
\text { Ensure consumers are not misled by vendors that } \\
\text { manufacture products with no health benefit and/or } \\
\text { risk of toxicity } \\
\text { Farmers that harvest crop and animals sources of } \\
\text { food and food ingredients that prevent disease } \\
\text { Develop and support alliances with stakeholders that } \\
\text { promote the commodities they grow } \\
\text { The foundation of the functional food industry } \\
\text { Utilise functional foods and nutraceutical products to } \\
\text { reduce risk factors for disease } \\
\text { Consumer beliefs, attitudes and behaviours provide } \\
\text { insight to stakeholders, which facilitate innovation } \\
\text { and sustained growth of the functional food industry }\end{array}$ \\
Producers & \\
Consumers &
\end{tabular}

Food and supplement companies realise the profitability of investing in the discovery, refinement and proof of efficacy of novel functional foods, functional food ingredients and nutraceuticals. By funding human clinical trials that explore and confirm their products' efficacy to reduce risk factors for disease, industry stakeholders can increase market penetration and product profitability via substantiating health claims. Given that mechanisms of action are important in obtaining health claims, industry stakeholders are not averse to funding human and animal trials that decipher, biochemically and physiologically, how their products induce a health benefit. Evidence of the food industry's interest in FFN can be found within the scientific literature, where companies have funded clinical and preclinical studies investigating the health benefits of functional foods and functional food ingredients ${ }^{(5-10)}$.

Scientists. Given the stringent regulations surrounding the approval of health claims for new FFN products, scientists from academia, government and industry represent an important component of the FFN marketplace. Well-designed preclinical and human clinical trials alongside sound scientific methodologies exist as the cornerstone in demonstrating the efficacy and mechanisms of action for how FFN reduce the prevalence of chronic disease. Thus, without scientific endeavour, few FFN would probably secure health claims and develop into profitable product lines. For example, stable-isotope methodologies have been instrumental in showing that plant sterols/ stanols (PS) decrease dietary cholesterol absorption and facilitate reductions in circulating LDL-cholesterol (LDL-C) levels. In addition, isotopic methods have been described as useful for measuring how FFN modulate bone metabolism for the prevention of osteoporosis ${ }^{(11)}$. Nutrigenomic techniques that identify the interaction between dietary constituents and gene regulation have been utilised to show that long-chain $n$ - 3 fatty acids decrease circulating TAG levels by modulating the expression of transcription factors that alter TAG synthesis and oxidation ${ }^{(12)}$. Finally, scientists use innovative technologies to discover biomarkers (biological identifiers that are utilised to identify the physiological state of an organism ${ }^{(13)}$ ) that suggest the progression or prevention of disease. For example, biomarkers exist as useful indicators for delineating the beneficial effects of FFN on the gastrointestinal microbiome and go beyond assessing fluctuations in specific bacterial populations. Microbe-derived metabolic intermediates and products, biomarkers within the mucin and epithelial cells, and chemical mediators that promote positive host-microbe and microbe-microbe interactions, can signify that FFN elicit positive effects on gastrointestinal microbiota and promote the health and wellbeing of the host $^{(14)}$. Recently, the effects of diet on circulating biomarkers of inflammation, including C-reactive protein and TNF- $\alpha$, have received significant attention amongst the scientific community as targets for investigating linkages between chronic disease and $\mathrm{FFN}^{(15)}$. It is evident 
that scientists' expertise and discoveries are important for sustained proof of efficacy and credibility within the FFN marketplace.

Scientists also provide expert opinions for decisions taken by the regulators on health claims in relation to FFN. This process ensures that robust science is utilised by regulators when formulating public policy around the availability of efficacious and safe FFN. In addition, scientists act as key opinion leaders and serve as liaisons of reliable science to industry, governments and the general population. Given that a significant proportion of research is exploratory, scientists are often on the forefront of revealing the medicinal value of foods, novel functional foods and/or nutraceuticals. Over the last 20 years, a surge has occurred in the construction of research institutions by industry as well as academia focused on discovery and proof of efficacy of FFN (Table 2). Research facilities, such as those listed in Table 2, facilitate scientific discovery by expediting FFN research and heightening accessibility of results to the FFN industry and the general public.

\section{Regulators}

Regulatory bodies including government institutions enforce laws surrounding the sale and health claim messages for FFN. Given that many FFN interact with biological pathways that modulate disease processes ${ }^{(16,17)}$, regulators take on the responsibility of ensuring that FFN are efficacious and safe for human consumption. Since the intended use of FFN is to prevent chronic disease, it is crucial that regulating agencies make certain that FFN demonstrate efficacy. The latter ensures that consumers are not misled by vendors that manufacture products with no health benefit and/or possible toxic effects.

Although numerous jurisdictions around the world have implemented guidelines for the approval of FFN-based health claims, considerable disparity exists between regulatory agencies concerning the level of evidence needed to support such claims. Enacted in 1991,
Japan's regulations under Food for Specified Health Uses (FOSHU) is considered among the most advanced, with eight categories of health claims ${ }^{(18)}$. As of 2007, 755 items had been approved for FOSHU status ${ }^{(18)}$. As of May 2007, 7557 functional foods received approval by China's Ministry of Health and State Food and Drugs Administration. Of the twenty-seven approved categories for health claims in China, four relate to disease prevention. The European Commission relies on the European Food Safety Authority (EFSA) to review the scientific evidence that supports the approval of health claims for FFN. Since 2007, EFSA has received over 268 applications for Article 14 - claims on disease risk reduction and child development or health. To date, EFSA has published over ninety Article 14 scientific opinions ${ }^{(19)}$ and the European Commission has authorised the use of seven disease reduction health claims ${ }^{(20)}$. In the USA, the Food and Drug Administration (FDA) has authorised twelve health claims under the Nutrition Education Labeling Act (NELA). Under the Food and Drug Administration Modernization Act, the FDA has received four health claim notifications ${ }^{(21-23)}$. For claims that do not meet the stringent health claim requirements put forth by the NELA, the FDA has also approved numerous qualified health claims under six disease categories ${ }^{(24)}$. Food Standards Australia New Zealand is considering the approval of nine health claims that relate food or food ingredients to a specific disease or disease biomarker ${ }^{(25)}$. Finally, Health Canada has sanctioned nine disease reduction and therapeutic claims ${ }^{(26)}$. Compared with other jurisdictions, regulatory agencies within Australia, New Zealand and Canada are relatively conservative when approving health claims for food and food ingredients that decrease the risk of disease. Nonetheless, all regulatory agencies discussed in the present review are focused on ensuring that consumers, within their respective jurisdictions, are protected against inefficacious and unsafe $\operatorname{FFN}^{(18,23,27-30)}$.

Table 2. Research facilities that investigate the biological effects of novel functional foods and nutraceuticals

\begin{tabular}{lll}
\hline Facility or research programme & Affiliation & Location \\
\hline $\begin{array}{l}\text { Richardson Centre for Functional Foods and Nutraceuticals } \\
\text { Human Nutraceutical Research Unit }\end{array}$ & $\begin{array}{l}\text { University of Manitoba } \\
\text { University of Guelph }\end{array}$ & $\begin{array}{l}\text { Winnipeg, Manitoba, Canada } \\
\text { Guelph, Ontario, Canada }\end{array}$ \\
Institute of Functional Foods and Nutraceuticals & Laval University & Laval, Quebec, Canada \\
Plant and Food Research & New Zealand Crown Corporation & New Zealand, Australia, USA \\
Functional Foods Research Centre & Oxford Brookes University & Oxford, UK \\
Nutritional Physiology Research Centre & University of South Australia & Adelaide, Australia \\
David H. Murdock Research Institute & North Carolina Research Campus & Kannapolis, NC, USA \\
Nestlé® Research Centre & Nestlé Research ${ }^{\text {TM }}$ & Lausanne, Switzerland \\
Danone Research Centre & Danone & Palaiseau, France \\
The Wageningen Specialized Research Centre & Danone & Wageningen, The Netherlands \\
The Nutricia Research Centre & Danone & Liverpool, UK \\
Unilever Global Research and Development Centres & Unilever & Shanghai, China \\
& & Bangalore, India \\
& & Vlaardingen, The Netherlands \\
& & Port Sunlight and Colworth, UK \\
\hline
\end{tabular}




\section{Producers}

Producers represent the farmers that harvest crop and animals sources of food and food ingredients that prevent disease. With scientists demonstrating the health benefits of FFN and/or regulators facilitating the approval of health claims for whole food or food derivatives that prevent disease, increased value is brought to commodities used in the production of FFN. Producers are also important in forming and directly supporting alliances that promote the commodities they grow. For example, given that Canada is a major producer of pulse crops, pulse growers have developed an alliance termed Pulse Canada. In 2006, Pulse Canada launched the Pulse Innovation Project, a funding initiative that brought together academia, industry and government to increase the market value of Canada's pulse crops. Recent research funded by the Pulse Innovation Project demonstrated that whole and fractionated yellow pea flours reduce postprandial glycaemic response, circulating insulin levels, insulin resistance and waistline fat deposition ${ }^{(31,32)}$. Results from the aforementioned studies were instrumental in securing profitable agreements between a Canadian raw pea flour producer and a USA-based processing and distribution company. As producers become familiar with the science that supports the use of their goods to prevent disease, producers become involved in research for value-added uses and technologies, as well as nutritional research.

\section{Consumers}

Ultimately it is consumer engagement that drives the FFN marketplace. As discussed previously, part of FFN allure amongst regulatory stakeholders is the notion that FFN can be used as tools to reduce healthcare costs associated with lifestyle-related diseases. Generally speaking, as consumers continue to take responsibility for their health and wellbeing, they become increasingly motivated to learn how FFN prevent chronic disease, promote longevity and reduce or delay pharmaceutical dependence. Nonetheless, stakeholders must remain cognizant of trends that suggest which FFN are acceptable amongst consumers. Dieroff $^{(33)}$ discusses consumer demand for beverages that deliver true evidence-based health benefits. In addition to consumers' growing gravitation toward 'natural' and 'organic' products within the USA, they are particularly motivated to adopt marine $n$ - 3 - and coenzyme Q10-fortified dairy products ${ }^{(33)}$. Unless industry, scientists, regulators and producers take notice of consumers' food preferences, novel food and food ingredients will fail as acceptable food products, despite their ability to prevent disease.

For that reason, the food industry continues to conduct research into market trends for current and future FFN and shows varying degrees of acceptance for individual FFN within different jurisdictions. For example, Europe's apprehension toward GM and irradiated foods is well known. Conversely, Cox et al. ${ }^{(34)}$ found acceptance amongst US consumers for GM oils containing long-chain $n$-3 fatty acids for use in breads and animal feed for the production of $n-3$ meat products. The notion that consumers are becoming increasingly wary of drugs and their understated side effects ${ }^{(35)}$ works in favour of the FFN industry. Biltekoff ${ }^{(36)}$ suggests that three paradoxes determine consumers' acceptance of healthy foods. First, the pleasure/health paradox implies that good-tasting foods are unhealthy and the taste of healthy foods is unfavourable. Second, the technology/nature paradox claims that although consumers accept the use of technological innovation to create healthy foods, they also fear technology and associate pure and natural foods with health and wellbeing. Finally, the innovation/nature paradox assumes that consumers seek solutions to health problems through innovation, while simplicity is a means of solving health problems. Depending on the global region, certain paradoxes impose more strongly on food beliefs and the acceptance of specific FFN. Grunert ${ }^{(37)}$ suggests that health is only one aspect of consumers' perception of food quality and competes with taste, convenience and naturalness. In summary, for the aforementioned stakeholders to secure buy-in amongst consumers, the FFN marketplace must consider consumer attitudes toward FFN within various jurisdictions to ensure that consumers accept FFN as vehicles for facilitating health.

What factors define future growth of the functional food and nutraceutical industry?

\section{Health claim evidence standardisation}

Health claims dramatically increase the marketability of novel FFN. As discussed previously, various jurisdictions have dedicated resources to regulating functional foodbased health claims. Although frustrating to manufacturers of FFN, allowance of claims that lack strong scientific evidence threatens the credibility of the FFN industry. In order for FFN to displace soaring healthcare costs, it is crucial for FFN to impose observable health benefits. The latter also ensures confidence in the FFN industry from consumers and the medical community.

The harmonisation of health claim messaging is especially important in the conditions of FFN market globalisation. Although regulations are in place, jurisdictions have highly variable expectations in the amount and type of scientific evidence required to validate health claims. For example, while the USA concluded that sufficient scientific evidence supports that fibre-containing grain products, fruits and vegetables prevent cancer, Health Canada has declared that the available scientific evidence is insufficient to support a health claim that implies an inverse relationship between fibre and cancer ${ }^{(38)}$. Health Canada cites inconsistent conclusions and methodological 
differences between studies as the primary reasons for refuting the aforementioned claim ${ }^{(38)}$. Given their demographic similarities and the fact that nutrient reference values and dietary guidelines such as the dietary reference intakes (DRI) and the 'Manual of Clinical Dietetics'(39), respectively, represent nutritional harmonisation between Canada and the USA, the divisive stance on FFN health claims between Canada and the USA is discouraging. Recently, the European Union and EFSA have been subject to criticism for being overly restrictive and denying a large proportion of applications requesting health claims on foods ${ }^{(40,41)}$. Although detailed analysis of methods used by regulatory agencies to interpret data that support or refute a health claim is beyond the scope of the present paper, it is important to emphasise that the cost of clinical research can be a prohibitive factor for companies to present well-designed, evidence-based research that truly supports a health claim for a novel food or ingredient. Instead, applications for health claims can rely heavily on pre-existing health claim language that flagrantly overemphasises limited data that companies may possess ${ }^{(42)}$. Moreover, because regulatory agencies are divisive in their interpretation of scientific evidence that supports or refutes the validity of FFN-derived health claims, the medical community can become disengaged from recommending FFN to patients. Lack of harmonisation of health claim messaging can also confuse consumers and the media as to which FFN are beneficial. Thus, standardisation and harmonisation of health claims for FFN are required for FFN market globalisation, to sustain consumer confidence and optimise partnerships between stakeholders within the FFN industry.

Small steps have been taken to harmonise health claims across jurisdictions. Representing the European Union, the establishment of EFSA is an example of imposing standards for FFN-associated health claims across member states. Similarly, Codex Alimentarius, a commission created by the FAO and the WHO to develop global food standards and guidelines, has published a guidance document outlining how evidence for food-based health claims should be substantiated ${ }^{(43)}$. However, The International Alliance of Dietary/Food Supplement Associations has criticised the Codex Alimentarius's 'Guidelines for Use of Nutrition and Health Claims', implicating it as imposing too much emphasis on human clinical interventions and not placing enough emphasis on large-scale observational studies when considering evidence for food-based health claims ${ }^{(44)}$. Observational studies are important sources of data especially for diseases including cancer, where incidence rates are difficult to predict. Nonetheless, given their size and time to completion, observational studies run the risk of accumulating confounders that lead to erroneous conclusions and, thus, study design, the sample population, data analysis, bias and statistical analysis should be carefully examined before definitive conclusions and subsequent health claims are established. It should be noted, however, that the Codex Alimentarius is not a regulatory agency. Instead, documents published by Codex Alimentarius serve as an international benchmark and reference point for regulatory agencies to begin implementing sovereign regulations for foods such as health claims.

Asia represents another major sector for FFN-regulatory harmonisation. However, countries that comprise Asia have only recently implemented inclusive RDA across the region. In 1997, The International Life Sciences Institute-Southeast Asia Region (ILSI SEA) held a workshop to discuss the logistics of harmonising RDA across Southeast Asia ${ }^{(45)}$. Discussions at the 1997 ILSI SEA workshop recommended that proposed RDA are comprised of two values, one that addresses nutritional deficiency and another to prevent chronic disease ${ }^{(45)}$. After 1997, an Ad Hoc Committee (SEA-RDAC) was formed consisting of members of local/national RDA committees from a number of Southeast Asian countries, namely Cambodia, Indonesia, Laos, Malaysia, Myanmar, Philippines, Singapore, Thailand and Vietnam. The SEA-RDAC has successfully implemented harmonised $\mathrm{RDA}^{(46)}$. Outside resources such as the $\mathrm{FAO} / \mathrm{WHO}$ Human Vitamin and Mineral Requirements were used to compose detailed scientific position papers for core nutrients and energy intake ${ }^{(46)}$. In addition, reference body weights for different populations were established and can be accessed by any country within Southeast Asia ${ }^{(46)}$. The harmonisation of RDA across Cambodia, Indonesia, Laos, Malaysia, Myanmar, Philippines, Singapore, Thailand and Vietnam sets the foundation for future consensus regarding FFN regulations. To date, we are unaware of any plans for Chinese or Japanese agencies to synchronise FFN regulations with other jurisdictions.

Overall, establishing consensus for FFN-derived health claims across jurisdictions would support the FFN industry by instilling confidence amongst consumers and the medical community for FFN to help mitigate the risk of disease. Nonetheless, collaborations between Canada and the USA as well as the establishment of EFSA and SEA-RDAC are indicative that the process of harmonisation has started.

\section{Overlap between functional food and pharmaceutical industries}

The health benefits of functional foods stem from the presence of bioactive components or nutraceuticals. Typically, nutraceuticals are components of food synthesised during their natural development and consumed in their native state or extracted and added to other food matrices. However, new technologies that modify or enhance the therapeutic properties of nutraceuticals are beginning to blur the line between what constitutes an FFN $v$. what constitutes a drug. For example, plant sterols, natural components of plant materials, and marine-derived $n-3$ fatty acids, EPA and DHA, are well known for their 
LDL-C- and TAG-lowering capabilities, respectively. However, LDL-C- and TAG-lowering properties of plant sterols and marine-derived fatty acids are exclusive to each component (Fig. 1). That is, plant sterols do not reduce TAG levels, while marine-derived $n-3$ fatty acids do not decrease circulating LDL-C levels. Thus, on their own, it is easy to classify plant sterols and marine-derived $n-3$ fatty acids as nutraceuticals. However, when the esterification of plant sterols to marine $n-3$ fatty acids creates a bioactive that facilitates reductions in circulating LDL-C and TAG that is equivalent to consuming each compound individually ${ }^{(47)}$, does the synthetic nature of the compound, alongside the food matrices to which it is housed, change its classification from an FFN to a drug? To cite another example, the identification of nutraceuticals has led to technologies that facilitate the production of synthetic analogues for natural compounds. For nutraceuticals that are extracted in relatively small quantities from parent foods, the synthetic production of bioactives can improve production efficiency. However, concerns over safety arise when synthetic analogues exhibit differences in molecular structure and enhanced efficacy when compared with native forms. While plant stanols, in nature, are hydrophobic compounds, Ebine et al. ${ }^{(48)}$ demonstrated that hamsters consuming diets containing synthetic hydrophilic plant stanol ester, disodium ascorbyl phytostanyl phosphates, at $0.71 \%$ reduced circulating cholesterol $(-34 \%)$ better than diets containing $1 \%$ natural hydrophobic plant stanols $(-14 \%)$. In addition, hamsters consuming synthetic plant stanol esters demonstrated a $42 \%$ reduction in TAG levels compared with controls, while no decreases in TAG levels were observed in hamsters consuming natural plant stanols ${ }^{(49)}$. In regulatory terms, the production of synthetic nutraceuticals elicits concerns over safety and distorts the traditional definition of FFN, which implies that foods and bioactives are of natural origin.

The intersect between functional foods and pharmaceutical agents is also demonstrated when evaluating the evidence that is utilised to establish the efficacy of a food containing a bioactive agent. For example, probiotics or prebiotics are consumed as means to modulating the gastrointestinal microbiome to facilitate a health benefit to the host. However, certain diseases of the gastrointestinal tract, such as Crohn's disease and ulcerative colitis, demonstrate perturbations in the diversity of gastrointestinal microflora compared with normal or healthy individuals ${ }^{(50-53)}$. Therefore, when a probiotic or prebiotic corrects a physiological function associated with a disruption in gastrointestinal microbial diversity, as seen in conditions of the alimentary tract, or alters the gastrointestinal microbial diversity such that it more closely resembles the microbiome of a healthy individual, should that proor prebiotic be characterised as a drug? This debate becomes more complex if healthy individuals do not benefit from consuming the same pre- or probiotic food or supplement. Given that functional foods are available in grocery stores and carry health claims that are directed at the general public, one could argue that the health benefits of functional foods should be attainable by the majority of the population. Although functional foods
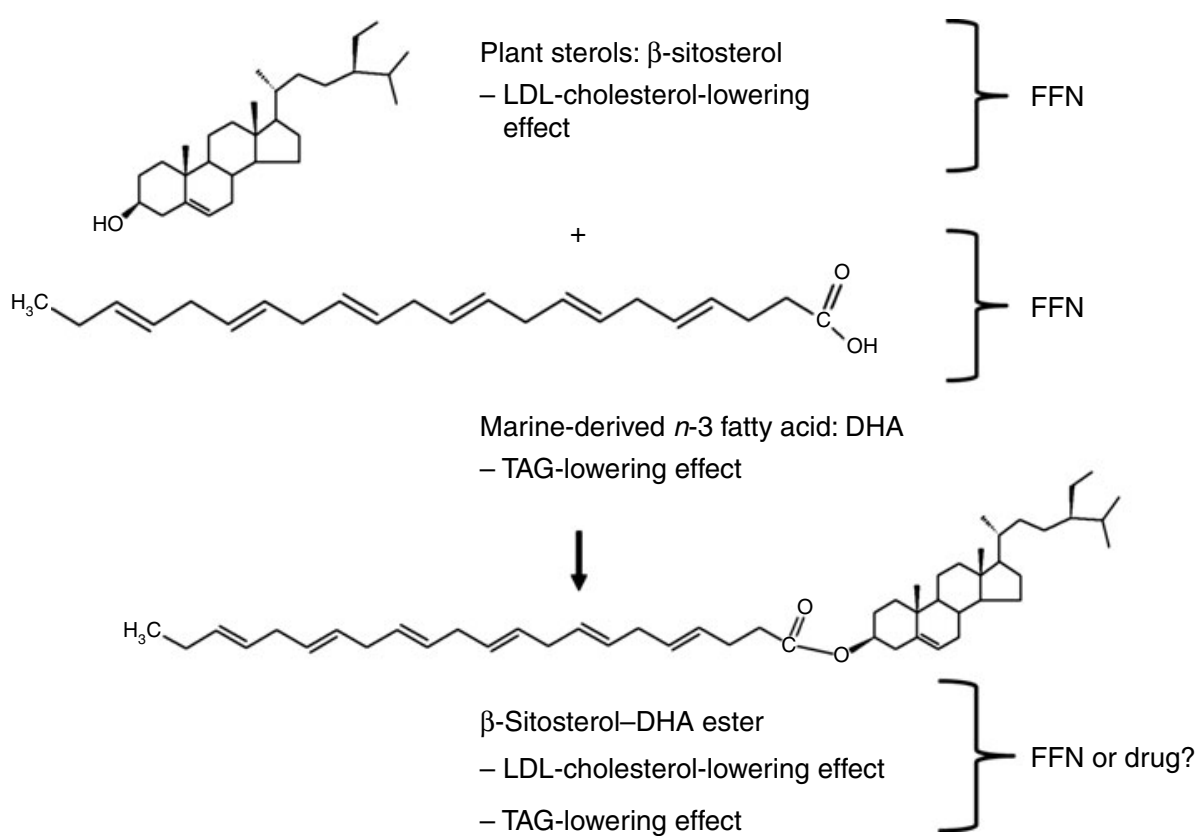

Fig. 1. On their own, the LDL-cholesterol (LDL-C)-lowering and TAG-lowering effects of plant sterols and marine-derived $n$-3 fatty acids are mutually exclusive and are individually classified as functional foods and nutraceuticals (FFN). However, esterification of plant sterols with marine $n$ - 3 fatty acids yields a synthetic molecule that induces combined LDL-C- and TAG-lowering efficacy. The production of efficacious synthetic bioactives from FFN facilitates new questions regarding their safety as well as their classification as FFN or drugs. 
are characterised as being of natural origin, regulatory agencies must decide how foods containing efficacious levels of bioactive ingredients are identified, as a food or drug, when the bioactive in question is only efficacious in the presence of disease.

Indeed, the pharmaceutical industry and regulatory agencies have begun classifying nutraceuticals extracted and consumed in capsule or tablet form as drugs. Lovaza ${ }^{\circledR}$, a highly concentrated encapsulated mixture of EPA and DHA produced by Pfizer, is classified as a prescription medication in the USA ${ }^{(17)}$. However, the FFN regulatory framework amongst jurisdictions has yet to fully address how to classify highly purified and/or modified/synthetic nutraceuticals within a food matrix. Given concerns over safety, regulatory agencies face new challenges for defining when foods and associated nutraceuticals remain 'functional foods' or become drugs.

\section{Are functional foods redefining nutrient requirements?}

Recommended nutrient intakes, and more recently, DRI, were established to provide the public with appropriate daily nutrient requirements to ensure proper physiological functionality and to prevent disorders secondary to nutrient deficiencies, such as rickets. However, with the cross-jurisdiction acknowledgement that functional foods and associated nutraceuticals can provide benefits that extend beyond the prevention of maladies associated with nutritional deficiencies, it can be argued that functional foods could redefine nutrient requirements. Moreover, historic assessments of diet indicate that levels of specific bioactive compounds, now characterised by modern science as nutraceuticals or natural health products, were consumed by our ancestors at much greater levels than currently consumed in Western diets. Levels of $n-3$ fats, fibres, PS and probiotics were much higher in diets of 10000 years ago than we consume today ${ }^{(54)}$. Thus, it can be argued that established values for ingesting FFN that elicit benefits beyond that of preventing nutrient deficiencies are the result of an evolutionary interaction between diet/bioactives and physiological function. The fact is that diets, especially in the Western hemisphere, have 'whitened' over the past century, dramatically shifting away from the consumption of FFN and becoming devoid in bioactives. This shift has contributed to an alarming present-day increase in the prevalence of lifestyle-related diseases, such as CVD and diabetes.

The aforementioned notion can be exemplified using the long-chain $n-3$ fatty acids, EPA and DHA, typically of marine origin. In developing the DRI for $n$ - 3 fatty acids, The National Academy of Sciences indicates that adults should consume 1.0-1.2 g $\alpha$-linolenic acid/d and specifies that the primary function for $\alpha$-linolenic acid is to serve as the precursor for endogenous synthesis of EPA and $\mathrm{DHA}^{(55)}$. However, the combined efficiency for converting $\alpha$-linolenic acid to EPA and DHA is less than $0.4 \%{ }^{(56)}$.
Although the structure/functional roles of EPA and DHA, including eye and brain development, are well known, research over the last decade consistently demonstrates the cardioprotective benefits of consuming 1-5g EPA and DHA/d, levels that can typically not be reached by consuming the recommended $1 \cdot 0-1 \cdot 2 \mathrm{~g} \alpha$-linolenic acid/d. Historic accounts demonstrate that marine-derived $n-3$ fatty acids were important dietary constituents during the course of human evolution, with research suggesting that dietary EPA and DHA were essential for hominin encephalisation $^{(57)}$. The aforementioned theory is further supported by the fact that, at higher levels of intake, marine-derived $n-3$ fatty acids modulate biological processes that facilitate the development of chronic disease such as serving as precursors to the synthesis of anti-inflammatory prostaglandins and acting as ligands for transcriptions factors that modulate the expression of genes facilitating TAG synthesis and fatty acid oxidation ${ }^{(12,58,59)}$. Simopoulos ${ }^{(60)}$ indicates that during the course of evolution the $n-6: n-3$ essential fatty acid ratio has dramatically shifted from $1: 1$ to 15-16:1, typical of a Western diet. In addition, based on the conversion efficiency of $\alpha$-linolenic acid to EPA and DHA, consumption of a balanced diet that incorporates a 1:1 n-6:n-3 essential fatty acid ratio would facilitate the synthesis of EPA and DHA to levels that would be cardioprotective. Populations consuming traditional diets that provide high levels of dietary EPA and DHA ${ }^{(61)}$ demonstrate lower levels of CVD. An analysis by Simopoulos ${ }^{(59)}$ showed that the combined $n-6: n-3$ in Europe and the USA is 50:1, compared with 12:1 and 1:1 amongst Japan and Greenland Inuit, respectively. The same analysis revealed that the incidence of cardiovascular-related mortality is 3.7 and 6.4 times less amongst the Japanese and Greenland Inuit compared with US and European populations. Thus, given that the cardioprotective roles of EPA and DHA probably stem from a co-evolutionary relationship where $n-3$ fatty acids represent a substantial constituent of the diet, it can be suggested that present dietary requirements for $n-3$ fatty acids are underestimated. Nutritional requirements should recommend higher intakes of $n$-3 fatty acids, reflecting their broad biological role for maintaining health and preventing disease.

PS serve as another example of the interaction between dietary bioactives and proper physiological function. Substantial research demonstrates 5-15\% LDL-C-lowering efficacy of consuming $1.5-2.5 \mathrm{~g} \mathrm{PS} / \mathrm{d}^{(62-64)}$. Mechanistically, dietary PS inhibit cholesterol absorption within the gastrointestinal tract. However, unlike $n-3$ fatty acids, no DRI exists for PS. Evolutionary accounts demonstrate that PS were probably consumed at $1 \mathrm{~g} / \mathrm{d}^{(65,66)}$. Thus, it is hypothesised that given PS historical prevalence within the diet, the evolution of physiological processes that facilitate cholesterol homeostasis, in part, reflects the presence of dietary $\mathrm{PS}^{(66)}$. The observation that PS act as agonists for transcription factors that increase the expression of ATP-binding cassette $G$ transporters within the intestinal 
epithelial membrane ${ }^{(67)}$ lends further support to a historical presence of PS within the diet. Although recent accounts demonstrate that $1.5-2.5 \mathrm{~g}$ PS/d are most efficacious for lowering LDL- $\mathrm{C}^{(62-64)}$, one must consider that, alongside less physical activity, modern diets provide higher density of energy and cholesterol as well as less fibre compared with diets that preclude the 20th century. Therefore, if PS efficacy stems from competitive inhibition of cholesterol absorption, it is not unreasonable that in order to help maintain cholesterol homeostasis, higher levels of PS must be present within modern diets compared with historical diets. Given levels of PS in ancestral diets and the presence of PS-gene interactions there is validity for PS to be designated an essential nutrient.

Given that physiological processes reflect the environmental landscape, including diet, over millions of years, it is reasonable that the sudden subtraction of certain dietary components has contributed to an increase in the incidence of disease. Evaluation of dietary guidelines demonstrates a disconnect between daily requirements for essential and non-essential nutrients and possible health benefits of FFN. If the incidence of disease can be prevented by consuming levels of FFN beyond what is recommended within DRI, it is logical that daily requirements for bioactive nutrients are underestimated.

\section{Conclusions}

The global functional food and nutraceutical industry represents a formidable initiative that continues to experience substantial growth. However, continued success of the FFN industry will rely on optimisation of roles and linkages between essential stakeholder groups. Food and supplement companies manufacture and distribute FFN, and provide funding for clinical trials that demonstrate that novel FFN are efficacious and safe. In addition to facilitating preclinical and clinical trials that prove FFN safety and efficacy as well as decipher mechanisms by which FFN benefit health, scientists formulate new hypotheses and act as key opinion leaders to ensure that sound, objective science regarding efficacious FFN is communicated to regulatory agencies and the public. Regulators introduce and enforce laws that make certain that the public is not misled by products that are unsafe and/or fail to elicit health benefits, while producers harvest crop and animal sources of food and food ingredients that prevent disease as well as form alliances which support commodity research and development. Finally, the end-users of FFN, consumers, drive the entire process chain by continuing to pay a premium price point for these products which in turn propels further FFN development, research and production. However, the lack of harmonisation between jurisdictions for approving FFN-based health claims, the blurred lines regarding the classification of FFN as foods or pharmaceuticals, and present challenges for developing dietary requirements that reflect therapeutic dosages of nutrients, all represent issues that can hinder future growth of the FFN industry. Functional foods of the future will continue in an upward trajectory so long as these fundamental issues are successfully addressed.

\section{Acknowledgements}

This research received no specific grant from any funding agency in the public, commercial or not-for-profit sectors.

C. P. F. M. and P. J. H. J. were responsible for conceptualising and writing the present paper.

Neither author has any conflicts of interest.

\section{References}

1. Agriculture and Agri-food Canada (2009) What are functional foods and nutraceuticals? http://www4.agr.gc.ca/AAFC-AAC/ display-afficher.do?id=1171305207040\&lang=eng (accessed 25 September 2011).

2. Tapsell LC, Williams P, Droulez V, et al (2005) Functional Foods for the Australian Industry: Definitions and Opportunities. Wollongong: National Centre of Excellence in Functional Foods.

3. Blandon J, Cranfield J \& Herson S (2007) Global market for functional foods and supplements. In Functional Food and Natural Health Product Issues: The Canadian and World Context. InFERG Working Paper no. 18. Guelph: International Food Economy Research Group, University of Guelph.

4. Blandon J, Cranfield J \& Herson S (2007) Market share of functional foods and natural health products by sales, 2003. In Functional Food and Natural Health Product Issues: The Canadian and World Context. InFERG Working Paper no. 18. Guelph: International Food Economy Research Group, University of Guelph.

5. Martin FP, Rezzi S, Pere-Trepat E, et al. (2009) Metabolic effects of dark chocolate consumption on energy, gut microbiota, and stress-related metabolism in free-living subjects. J Proteome Res 8, 5568-5579.

6. Rideout TC, Chan YM, Harding SV, et al. (2009) Low and moderate-fat plant sterol fortified soymilk in modulation of plasma lipids and cholesterol kinetics in subjects with normal to high cholesterol concentrations: report on two randomized crossover studies. Lipids Health Dis 8, 45.

7. Guillemard E, Tondu F, Lacoin F, et al. (2010) Consumption of a fermented dairy product containing the probiotic Lactobacillus casei DN-114001 reduces the duration of respiratory infections in the elderly in a randomised controlled trial. $\mathrm{BrJ}$ Nutr 103, 58-68.

8. Harding SV, Zhao HL, Marinangeli CP, et al. (2009) Red algal cellular biomass lowers circulating cholesterol concentrations in Syrian golden hamsters consuming hypercholesterolaemic diets. BrJ Nutr 102, 842-847.

9. Philippe D, Favre L, Foata F, et al. (2011) Bifidobacterium lactis attenuates onset of inflammation in a murine model of colitis. World J Gastroenterol 17, 459-469.

10. De Bruin EA, Rowson MJ, Van Buren, et al. (2011) Black tea improves attention and self-reported alertness. Appetite 56, $235-240$

11. Weaver CM \& Liebman M (2002) Biomarkers of bone health appropriate for evaluating functional foods designed to reduce risk of osteoporosis. BrJ Nutr $\mathbf{8 8}$, Suppl. 2, S225-S232. 
12. Davidson MH (2006) Mechanisms for the hypotriglyceridemic effect of marine omega-3 fatty acids. Am J Cardiol 98, $27 \mathrm{i}-33 \mathrm{i}$.

13. Atkinson AJ, Magnuson WG, Colburn WA, et al. (2001) Biomarkers and surrogate endpoints: preferred definitions and conceptual framework. Clin Pharmacol Ther 69, 89-95.

14. Oozeer R, Rescigno M, Ross RP, et al. (2010) Gut health: predictive biomarkers for preventive medicine and development of functional foods. Br J Nutr 103, 1539-1544.

15. Puglisi MJ \& Fernandez ML (2008) Modulation of C-reactive protein, tumor necrosis factor- $\alpha$, and adiponectin by diet, exercise, and weight loss. J Nutr 138, 2293-2296.

16. Marinangeli CP \& Jones PJ (2010) Plant sterols, marinederived omega- 3 fatty acids and other functional ingredients: a new frontier for treating hyperlipidemia. Nutr Metab (Lond) 7, 76.

17. Marinangeli CP \& Jones PJ (2010) Functional food ingredients as adjunctive therapies to pharmacotherapy for treating disorders of metabolic syndrome. Ann Med 42, 317-333.

18. Yamada K, Sato-Mito N, Nagata J, et al. (2008) Health claim evidence requirements in Japan. J Nutr 138, 1192S-1198S.

19. European Food and Safety Agency (2011) Claims on disease risk reduction and child development or health under Article 14. http://www.efsa.europa.eu/en/topics/topic/article14. htm (accessed 22 January 2012).

20. European Commission (2012) EU Register on nutrition and health claims. http://ec.europa.eu/nuhclaims/?event=search $\& \mathrm{CFID}=169885 \&$ CFTOKEN $=77733 \mathrm{dc} 16 \mathrm{~b} 564 \mathrm{cec}-8 \mathrm{~B} 974 \mathrm{E} 68$ A55E-BCBD-81A1575D7393A10C\&jsessionid=93121b711 d2370b2022b8045d657a69522a4TR (accessed 15 July 2012).

21. US Food and Drug Administration (2003) Claims that can be made for conventional foods and dietary supplements. http://www.fda.gov/food/labelingnutrition/labelclaims/ucm 111447.htm (accessed 21 February 2011).

22. US Food and Drug Administration (2009) Guidance for industry: a food labeling guide: appendix $\mathrm{C}$ - health claims. http://www.fda.gov/Food/GuidanceComplianceRegulatory Information/GuidanceDocuments/FoodLabelingNutrition/ FoodLabelingGuide/ucm064919.htm (accessed 21 February 2011).

23. Hasler CM (2008) Health claims in the United States: an aid to the public or a source of confusion? J Nutr 138, 1216S-1220S.

24. US Food and Drug Administration (2011) Summary of qualified health claims subject to enforcement discretion. http://www. fda.gov/Food/LabelingNutrition/LabelClaims/Qualified HealthClaims/ucm073992.htm (accessed 22 January 2012).

25. Food Standards Australia New Zealand (2012) Call for submissions - proposal P293: nutrition, bealth and related claims, vol. 4-12. Canberra and Wellington: Food Standards Australia New Zealand.

26. Health Canada (2010) Health claim assessments. http://www. hc-sc.gc.ca/fn-an/label-etiquet/claims-reclam/assess-evalu/ index-eng.php (accessed 11 July 2012).

27. L'Abbe MR, Dumais L, Chao E, et al. (2008) Health claims on foods in Canada. J Nutr 138, 1221S-1227S.

28. Tapsell LC (2008) Evidence for health claims: a perspective from the Australia-New Zealand region. I Nutr 138, 1206S-1209S.

29. Yang Y (2008) Scientific substantiation of functional food health claims in China. J Nutr 138, 1199S-1205S.

30. European Commission (2007) Corrigendum to regulation (EC) no. 1924/2006 of the European Parliament and of the Council of 20 December 2006 on nutrition and health claims made on foods. In Official Journal of the European Union, pp. L12/3-L12/18. Brussels: Publications Office.

31. Marinangeli CP \& Jones PJ (2011) Whole and fractionated yellow pea flours reduce fasting insulin and insulin resist- ance in hypercholesterolaemic and overweight human subjects. Br J Nutr 105, 110-117.

32. Marinangeli CP, Kassis AN \& Jones PJ (2009) Glycemic responses and sensory characteristics of whole yellow pea flour added to novel functional foods. J Food Sci $\mathbf{7 4}$, S385-S389.

33. Dieroff C (2011) Beverage trends: consumers want it all. In Prepared Foods, vol. February, pp. 49-55. Deerfield, IL: Business News Publishing Co.

34. Cox DN, Evans G \& Lease HJ (2011) The influence of product attributes, consumer attitudes and characteristics on the acceptance of: (1) novel bread and milk, and dietary supplements and (2) fish and novel meats as dietary vehicles of long chain omega 3 fatty acids. Food Qual Preference 22, 205-212.

35. Olsen AK \& Whalen MD (2009) Public perceptions of the pharmaceutical industry and drug safety: implications for the pharmacovigilance professional and the culture of safety. Drug Saf 32, 805-810.

36. Biltekoff C (2010) Consumer response: the paradoxes of food and health. Ann N Y Acad Sci 1190, 174-178.

37. Grunert KG (2010) European consumers'acceptance of functional foods. Ann N Y Acad Sci 1190, 166-173.

38. Health Canada (2001) Health Canada scientific summary on the U.S. health claim on fibre-containing grain products, fruits and vegetables and cancer. http://www.hc-sc.gc.ca/ fn-an/label-etiquet/claims-reclam/assess-evalu/fibre_cancereng.php (accessed 22 January 2012).

39. Chicago Dietetic Association, South Suburban Dietetic Association (Ill.) \& Dietitians of Canada (2000) Manual of Clinical Dietetics, 6th ed. Chicago, IL: American Dietetic Association.

40. Starling S (2011) EU researchers revolted as EFSA clears health claims vault. http://www.nutraingredients.com/ Regulation/EU-researchers-revolted-as-EFSA-clears-health-claims-vault (accessed 2 October 2011).

41. Biesalski HK, Aggett PJ, Anton R, et al. (2011) 26th Hohenheim Consensus Conference, September 11, 2010 Scientific substantiation of health claims: evidence-based nutrition. Nutrition 27, S1-S20.

42. Starling S (2011) NDA panellist: angry academics would come to same conclusions if on our panel. http://www. nutraingredients.com/Regulation/NDA-panellist-Angry-academics-would-come-to-same-conclusions-if-on-our-panel (accessed 2 October 2011).

43. Codex Alimentarius (1997) Guidelines for Use of Nutrition and Health Claims, CAC/GL 23-1997. Rome: Codex Alimentarius.

44. Daniells S (2008) IADSA keeps pressure on Codex over science for health claims. http://www.nutraingredients. com/Regulation/IADSA-keeps-pressure-on-Codex-over-science-for-health-claims (accessed 11 September 2011).

45. Florentino RF (1997) The ILSI SEA Regional Forum and Workshop on recommended dietary allowances: scientific basis and future directions. Asia Pac J Clin Nutr 7, 96-101.

46. Barba CV \& Cabrera MI (2008) Recommended dietary allowances harmonization in Southeast Asia. Asia Pac J Clin Nutr 17, Suppl. 2, 405-408.

47. Demonty I, Chan YM, Pelled D, et al. (2006) Fish-oil esters of plant sterols improve the lipid profile of dyslipidemic subjects more than do fish-oil or sunflower oil esters of plant sterols. Am J Clin Nutr 84, 1534-1542.

48. Ebine N, Jia X, Demonty I, et al. (2005) Effects of a water-soluble phytostanol ester on plasma cholesterol levels and red blood cell fragility in hamsters. Lipids $\mathbf{4 0}$, $175-180$. 
49. Ebine N, Demonty I, Jia X, et al. (2006) Plant stanol ascorbate esters reduce body weight gain through decreased energy absorption in hamsters. Int $J$ Obes (Lond) 30, 751-757.

50. Macfarlane GT, Furrie E \& Macfarlane S (2004) Bacterial milieu and mucosal bacteria in ulcerative colitis. Novartis Found Symp 263, 57-70, 211-218.

51. Negroni A, Costanzo M, Vitali R, et al. (2012) Characterization of adherent-invasive Escherichia coli isolated from pediatric patients with inflammatory bowel disease. Inflamm Bowel Dis 18, 913-924.

52. Ott SJ, Musfeldt M, Wenderoth DF, et al. (2004) Reduction in diversity of the colonic mucosa associated bacterial microflora in patients with active inflammatory bowel disease. Gut 53, 685-693.

53. Sepehri S, Kotlowski R, Bernstein CN, et al. (2007) Microbial diversity of inflamed and noninflamed gut biopsy tissues in inflammatory bowel disease. Inflamm Bowel Dis 13, $675-683$.

54. Jew S, AbuMweis SS \& Jones PJ (2009) Evolution of the human diet: linking our ancestral diet to modern functional foods as a means of chronic disease prevention. $J$ Med Food 12, 925-934.

55. Otten JJ, Hellwig JP \& Meyers LD (2006) Dietary Reference Intakes: The Essential Guide to Nutrient Requirements. Washington, DC: National Academies Press.

56. Hussein N, Ah-Sing E, Wilkinson P, et al. (2005) Long-chain conversion of $\left[{ }^{13} \mathrm{C}\right]$ linoleic acid and $\alpha$-linolenic acid in response to marked changes in their dietary intake in men. J Lipid Res 46, 269-280.

57. Cunnane SC, Plourde M, Stewart K, et al. (2007) Docosahexaenoic acid and shore-based diets in hominin encephalization: a rebuttal. Am J Hum Biol 19, 578-581.
58. Balk EM, Lichtenstein AH, Chung M, et al. (2006) Effects of omega-3 fatty acids on serum markers of cardiovascular disease risk: a systematic review. Atherosclerosis 189, 19-30.

59. Simopoulos AP (2002) Omega-3 fatty acids and cardiovascular disease: the epidemiological evidence. Environ Health Prev Med 6, 203-209.

60. Simopoulos AP (2006) Evolutionary aspects of diet, the omega-6/omega-3 ratio and genetic variation: nutritional implications for chronic diseases. Biomed Pharmacother 60 , 502-507.

61. Elmadfa I \& Kornsteiner M (2009) Dietary fat intake - a global perspective. Ann Nutr Metab 54, Suppl. 1, 8-14.

62. Abumweis SS, Barake R \& Jones PJ (2008) Plant sterols/ stanols as cholesterol lowering agents: a meta-analysis of randomized controlled trials. Food Nutr Res 52, 1811.

63. Demonty I, Ras RT, van der Knaap HC, et al. (2009) Continuous dose-response relationship of the LDL-cholesterollowering effect of phytosterol intake. J Nutr 139, 271-284.

64. Berger A, Jones PJ \& Abumweis SS (2004) Plant sterols: factors affecting their efficacy and safety as functional food ingredients. Lipids Health Dis 3, 5.

65. Jenkins DJ, Kendall CW, Marchie A, et al. (2003) The Garden of Eden - plant based diets, the genetic drive to conserve cholesterol and its implications for heart disease in the 21st century. Comp Biochem Physiol A Mol Integr Physiol 136, 141-151.

66. Jenkins DJ \& Kendall CW (2006) The garden of Eden: plantbased diets, the genetic drive to store fat and conserve cholesterol, and implications for epidemiology in the 21st century. Epidemiology 17, 128-130.

67. Plat J, Nichols JA \& Mensink RP (2005) Plant sterols and stanols: effects on mixed micellar composition and LXR (target gene) activation. J Lipid Res 46, 2468-2476. 